\title{
Prediction of Early Periprosthetic Joint Infection After Total Hip Arthroplasty
}

\author{
Erik Bülow $\mathbb{D}^{1,2}$, Ute Hahn ${ }^{3,4}$, Ina Trolle Andersen ${ }^{3}$, Ola Rolfson ${ }^{1,2}$, \\ Alma B Pedersen $\left(1 D^{3,5}\right.$, Nils P Hailer ${ }^{6}$
}

'The Swedish Arthroplasty Register, Centre of Registers Västra Götaland, Gothenburg, Sweden; ${ }^{2}$ Department of Orthopaedics, Institute of Clinical Sciences, The Sahlgrenska Academy, University of Gothenburg, Gothenburg, Sweden; ${ }^{3}$ Department of Clinical Epidemiology, Aarhus University Hospital, Aarhus, Denmark; ${ }^{4}$ Department of Mathematics, Aarhus University, Aarhus, Denmark; ${ }^{5}$ Department of Clinical Medicine, Aarhus University, Aarhus, Denmark;

${ }^{6}$ Department of Surgical Sciences/Orthopaedics, Uppsala University Hospital, Uppsala, Sweden

Correspondence: Erik Bülow, The Swedish Arthroplasty Register, Centre of Registers Västra Götaland, Gothenburg, SE-4I 3 45, Sweden, Tel +46 7008234 28, Email erik.bulow@gu.se

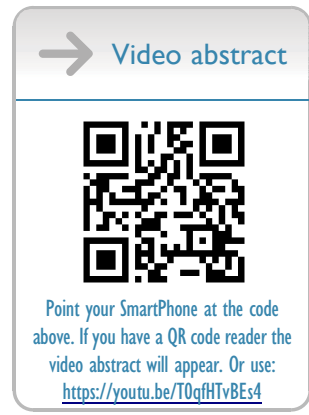

Purpose: To develop a parsimonious risk prediction model for periprosthetic joint infection (PJI) within 90 days after total hip arthroplasty (THA).

Patients and Methods: We used logistic LASSO regression with bootstrap ranking to develop a risk prediction model for PJI within 90 days based on a Swedish cohort of 88,830 patients with elective THA 2008-2015. The model was externally validated on a Danish cohort with 18,854 patients.

Results: Incidence of PJI was $2.45 \%$ in Sweden and $2.17 \%$ in Denmark. A model with the underlying diagnosis for THA, body mass index (BMI), American Society for Anesthesiologists (ASA) class, sex, age, and the presence of five defined comorbidities had an area under the curve (AUC) of 0.68 (95\% CI: 0.66 to 0.69) in Sweden and 0.66 (95\% CI: 0.64 to 0.69) in Denmark. This was superior to traditional models based on ASA class, Charlson, Elixhauser, or the Rx Risk V comorbidity indices. Internal calibration was good for predicted probabilities up to $10 \%$.

Conclusion: A new PJI prediction model based on easily accessible data available before THA was developed and externally validated. The model had superior discriminatory ability compared to ASA class alone or more complex comorbidity indices and had good calibration. We provide a web-based calculator (https://erikbulow.shinyapps.io/thamortpred/) to facilitate shared decision making by patients and surgeons.

Keywords: prediction model, total hip arthroplasty, orthopaedics, clinical decision-making tool, web calculator, external validation

\section{Introduction}

Periprosthetic joint infection (PJI) is one of the most devastating early complications after total hip arthroplasty (THA), mainly due to its association with increased mortality, re-operation risk, long-term antibiotic treatment, often persistently impaired quality of life, and socioeconomic consequences. ${ }^{1-4}$ Many risk factors for the development of PJI are identified, with anaemia, diabetes, and obesity being important, to some extent modifiable risk factors, whereas advanced age and male sex are examples of non-modifiable risk factors. ${ }^{5-17}$

Several attempts have been made at developing prognostic individualized risk prediction models for clinical usage, with the Universal American College of Surgeons National Surgical Quality Improvement Program (ACS NSQIP) Surgical Risk Calculator being one of the first. ${ }^{18,19}$ Additional models have since been derived on US or Australian cohorts, but none has gained general acceptance. ${ }^{19-21}$ This is mainly due to often limited, sometimes single-center based samples, low discriminating ability or sub-optimal calibration of the prediction models, or the absence of external model validation. 
We therefore aimed to develop a prognostic prediction model of PJI within 90 days after THA, a commonly used period to clinically define early PJI. ${ }^{14}$ We based the model on easily accessible data that are available pre-operatively in the setting of clinical decision-making. Furthermore, we aimed to validate the model both internally in Sweden, as well as externally on a Danish national cohort.

\section{Patients and Methods}

The derivation cohort was based on patients from an updated linkage database including the Swedish Hip Arthroplasty Register (SHAR). The included registers and linkage process has been described previously. ${ }^{22}$ We included all primary elective THAs performed in Sweden 2008-2015. Only the last operated hip was considered for patients with bilateral THA. ${ }^{23}$ Patients with missing information on the American Society of Anesthesiologists (ASA) class, or an ASA class of IV and above, were excluded, as were patients with missing information on body mass index (BMI) or a measurement above 50. Patients with missing information on educational levels or the type of Hospital were also excluded, as were persons under the age of 18 .

In addition to the Swedish derivation cohort, a Danish sample was used for external model validation. Danish THA patients were operated from 2016 until the last of September 2018. This period was chosen due to availability of information on ASA and BMI, and the possibility for all patients to have a minimum of 90 days of follow up. As in Sweden, data were linked from several national registers based on each patients unique identity number: ${ }^{24}$ DHR as a quality register for THA ${ }^{25}$ the Danish National Patient Register with comorbidity data from all hospitals, ${ }^{26}$ and educational level and civil status from Statistics Denmark. ${ }^{27}$ As patients with PJI in the Swedish cohort were of age 35 to 99 years, we chose to validate the model for this age span only.

\section{Definitions of Covariates and Outcomes}

Comorbidities were first identified by ICD-10 codes from each countries' national patient register during the year before surgery. Individual conditions were grouped, first according to Elixhauser and Charlson, and then again into broader, clinically relevant, categories. Comorbidities observed for less than 10 patients with or without PJI, however, were not considered as potential predictors, to reduce the risk of overfitting the model to spurious events.

PJI was defined by the occurrence of relevant ICD-10 or procedural Nordic Medico-Procedural Committee (NOMESCO)-codes recorded in the national patient registers for hospital episodes within 90 days after THA (Table 1), or if the patient was reported to SHAR or DHR as re-operated due to PJI within the same time frame.

\section{Model Development}

We based a statistical variable selection procedure on bootstrap ranking combined with logistic least absolute shrinkage and selection operator (LASSO) regression, in a way previously described and illustrated by Garland et al. ${ }^{28}$ The distilled predictors were then used in a multiple main effects logistic regression model, our "main model." A more parsimonious "reduced model" was built by keeping only the most influential predictors. Those two models were then compared to univariable prediction models based on either ASA class, the Charlson, Elixhauser, or the Rx Risk V comorbidity indices, ${ }^{29,30}$ as well as multivariable models combining those measures with age, sex and BMI.

Table I Codes Identifying Periprosthetic Joint Infection (PJI) if Recorded in the Swedish and Danish National Patient Registers Within 90 Days After Total Hip Arthroplasty (THA)

\begin{tabular}{|l|l|}
\hline Classification & Codes \\
\hline ICD-I0 & $\begin{array}{l}\text { M000, M000F, M00I, M002, M002F, M008, M008F, M009, M009F, M860F, M86IF, M866, M866F, T8I4, T845, T845F, T845X, T847, } \\
\text { T847F }\end{array}$ \\
\hline NOMESCO & NFS09, NFSI9, NFS29, NFS39, NFS49, NFS59, NFS99 \\
\hline
\end{tabular}




\section{Model Validation}

Receiver operating characteristic (ROC) curves and their corresponding areas under the curve (AUC) were used to describe discrimination with 95\% confidence intervals (CI) for each model. Predicted probabilities and observed proportions were also plotted with $95 \%$ confidence bands to graphically assess model calibration. ${ }^{31}$ Coefficients of determination were estimated by Nagelkerke's $R^{2}$ for each model, as was the fraction of new information for each model compared to the model with lowest $R^{2} .^{32}$

The model was externally validated on the Danish cohort, both with the coefficient estimates from the Swedish cohort, and with new estimates based on the Danish cohort. The reference models, with adopted coefficient estimates, were also applied to the Danish cohort for comparison.

\section{Statistical Tools}

We used R version 4.1 (R Foundation for Statistical Computing, Vienna, Austria) with significant packages coder, ${ }^{33}$ decoder, furrr, pROC, rms, tidymodels and tidyverse. We build a shiny-based web calculator to aid clinical usage (https://erikbulow. $\underline{\text { shinyapps.io/thamortpred/) and made all R scripts publicly available (https://doi.org/10.5281/zenodo.5914599). }}$

\section{Ethical Approval}

Ethical approval for this study was obtained from the Regional Ethical Review Board in Gothenburg (360-13). The study was also approved by the Danish Data Protection Agency (journal number 2015-57-0002) and recorded at Aarhus University (journal number 2016-051-000001).

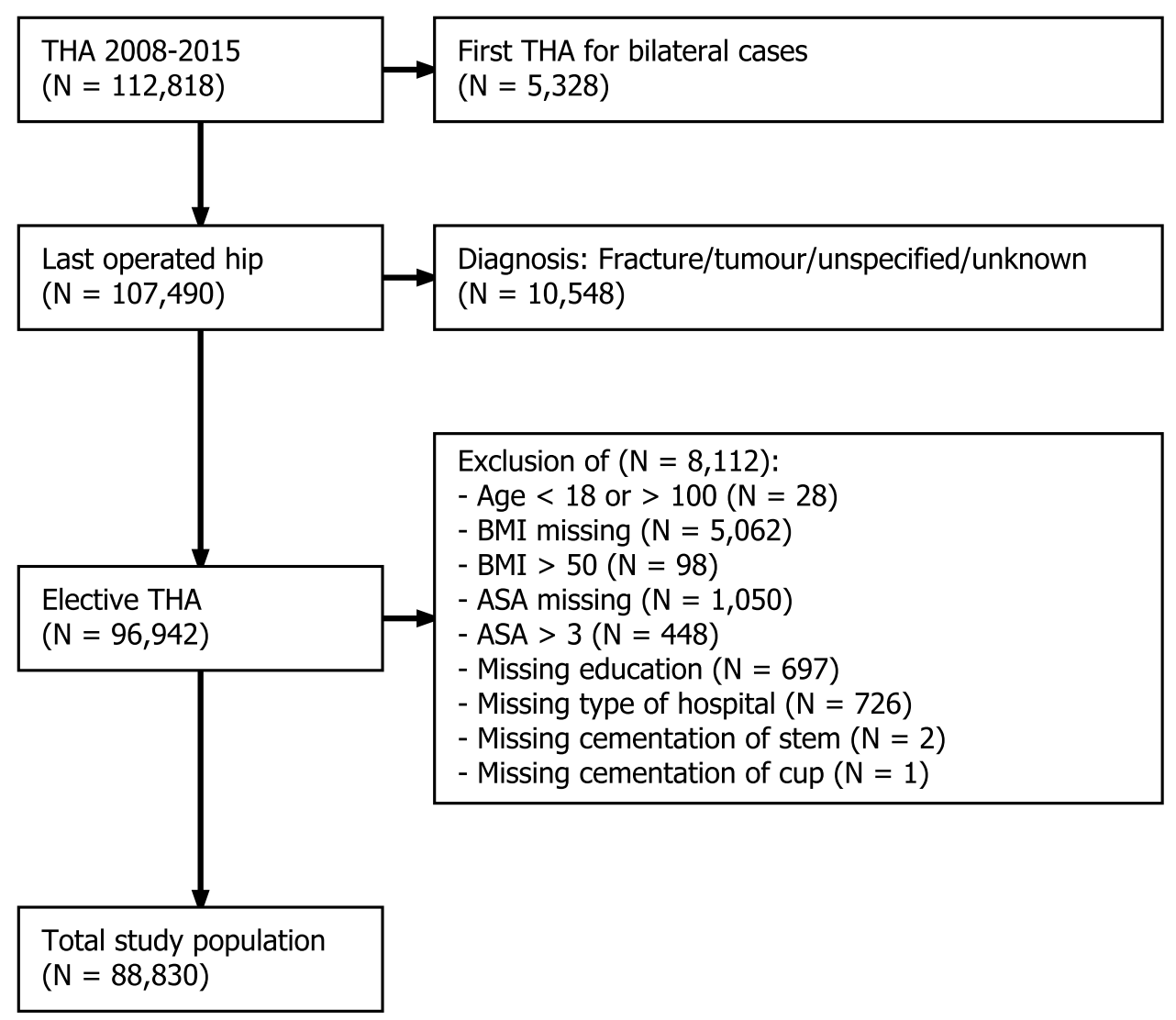

Figure I Flowchart with inclusion criteria and number of patients. Data from the Swedish Hip Arthroplasty Register were used for model derivation and internal validation. Abbreviations: THA, total hip arthroplasty; BMI, body mass index; ASA, American Society for Anesthesiologists classification. 


\section{Results}

We included 88,830 patients in the derivation cohort from Sweden (Figure 1). 2.45\% $(\mathrm{n}=2173)$ of these developed a PJI within 90 days. In addition, 18,854 patients were included in the external validation cohort from Denmark (Figure 2). $2.2 \%(\mathrm{n}=410)$ of these developed a PJI within 90 days (Table 2$)$.

ROC-curves (Figure 3) and AUC-values (Figure 4) were very similar for the main model and a reduced model, based on the underlying diagnosis for THA, BMI, ASA class, sex, age, and the presence of arrhythmia, CNS disease, fluid electrolyte disorders, liver disease or lung and airways disease. For simplicity, we thus focused on the reduced model, with estimated coefficient values listed in Table 3.

The univariable model with the Charlson comorbidity index had the lowest AUC and Nagelkerke's $R^{2}=0.73$, and compared to this model, the fraction of new information for the reduced model was $84 \%$ (Table 4 ). The reduced model had good internal calibration for predictions up to $10 \%$ (Figure 5). Estimated probability density curves for patients with

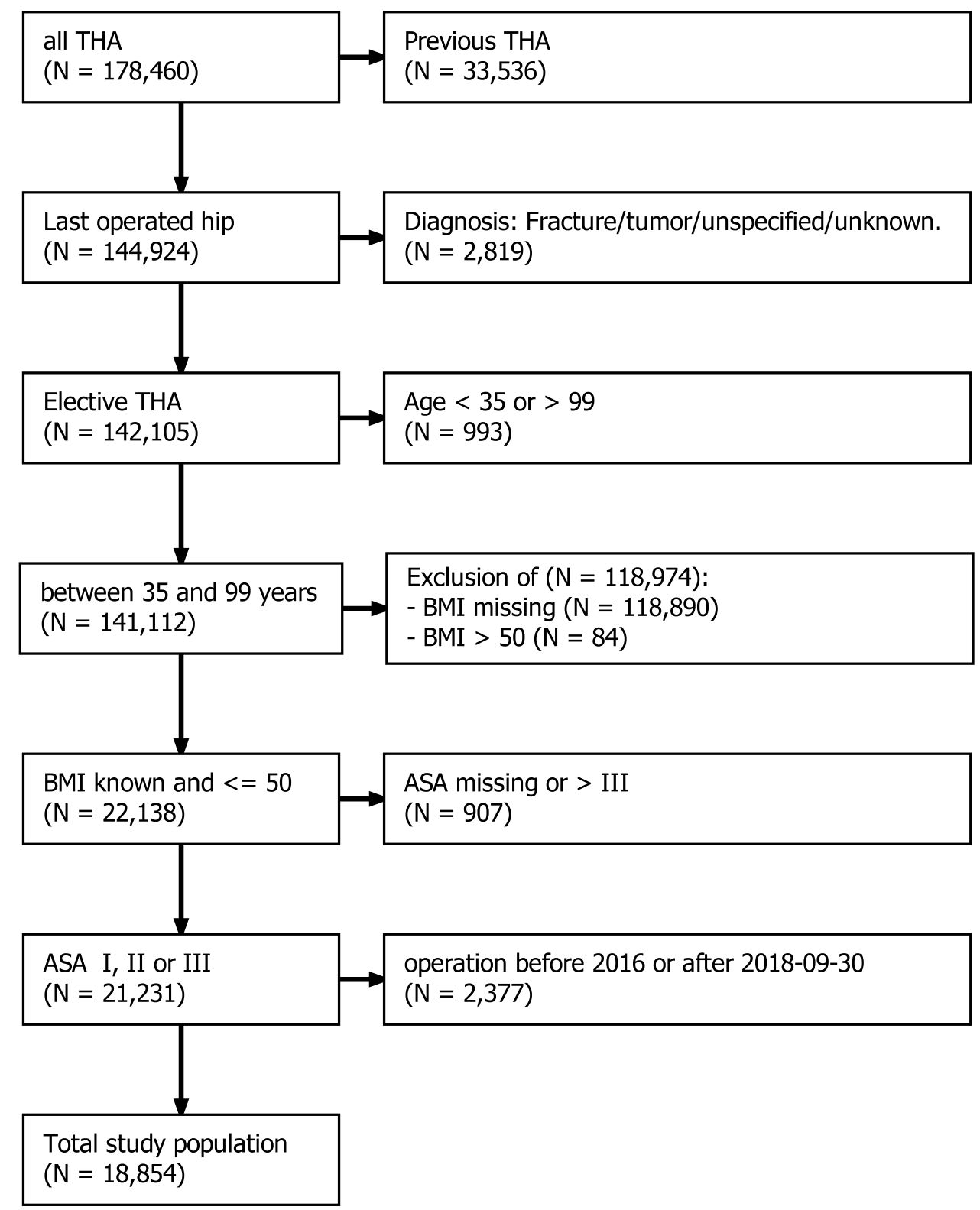

Figure 2 Flowchart with inclusion criteria and number of patients. Data from the Danish Hip Arthroplasty Register were used for external model validation. Abbreviations: THA, total hip arthroplasty; BMI, body mass index; ASA, American Society for Anesthesiologists classification. 
Table 2 Patient Characteristics in Sweden 2008-2015 and Denmark 2016 to September 2018

\begin{tabular}{|c|c|c|c|c|c|}
\hline Variable & Level & $\begin{array}{r}\text { SE PJI } \\
\text { n (\%) }\end{array}$ & $\begin{array}{r}\text { SE Total } \\
\text { n (\%) }\end{array}$ & $\begin{array}{r}\text { DK PJI } \\
\text { n (\%) }\end{array}$ & $\begin{array}{r}\text { DK Total } \\
\text { n (\%) }\end{array}$ \\
\hline Total & & 2173 & 88,830 & 410 & 18,854 \\
\hline \multirow[t]{5}{*}{ Age } & $<50$ & $104(4.8)$ & $4645(5.2)$ & $9(2.2)$ & $880(4.7)$ \\
\hline & $50-<60$ & $231(10.6)$ & $11,812(13.3)$ & $45(11.0)$ & $2460(13.0)$ \\
\hline & $60-<70$ & $609(28.0)$ & 28,981 (32.6) & $112(27.3)$ & $5226(27.7)$ \\
\hline & $70-<80$ & $776(35.7)$ & $30,386(34.2)$ & I64 (40.0) & $720 \mathrm{I}(38.2)$ \\
\hline & $80+$ & $453(20.8)$ & $13,006(14.6)$ & $80(19.5)$ & $3087(16.4)$ \\
\hline \multirow[t]{2}{*}{ Sex } & Female & $1062(48.9)$ & $50,151(56.5)$ & $209(5 \mathrm{I} .0)$ & 10,915 (57.9) \\
\hline & Male & IIII (5I.I) & $38,679(43.5)$ & $20 \mathrm{I}(49.0)$ & $7939(42.1)$ \\
\hline \multirow[t]{4}{*}{ BMI } & Under/normal weight & $487(22.4)$ & 29,431 (33.1) & I0I (24.6) & $6847(36.3)$ \\
\hline & Overweight & $863(39.7)$ & $38,143(42.9)$ & $152(37.1)$ & $7416(39.3)$ \\
\hline & Class I obesity & $531(24.4)$ & $16,224(18.3)$ & $86(21.0)$ & $3347(17.8)$ \\
\hline & Class II-III obesity & $292(13.4)$ & $5032(5.7)$ & $71(17.3)$ & $1244(6.6)$ \\
\hline \multirow[t]{3}{*}{ ASA class } & I & $302(13.9)$ & $21,087(23.7)$ & $44(10.7)$ & 3942 (20.9) \\
\hline & II & $1228(56.5)$ & $52,798(59.4)$ & $253(6 \mid .7)$ & II,578 (6I.4) \\
\hline & III & $643(29.6)$ & $14,945(16.8)$ & $113(27.6)$ & $3334(17.7)$ \\
\hline \multirow[t]{5}{*}{ Diagnosis } & Primary osteoarthritis & $1766(81.3)$ & $78,578(88.5)$ & $329(80.2)$ & $15,926(84.5)$ \\
\hline & Secondary osteoarthritis & $232(10.7)$ & $527 \mid(5.9)$ & $57(13.9)$ & $1838(9.7)$ \\
\hline & Sequelae after childhood hip disease & $4 \mid(1.9)$ & $1862(2.1)$ & $*$ & $626(3.3)$ \\
\hline & $\begin{array}{l}\text { Avascular necrosis of the femoral head } \\
\text { (AVN) }\end{array}$ & $77(3.5)$ & $1895(2.1)$ & $15(3.7)$ & $318(1.7)$ \\
\hline & Inflammatory joint disease & $57(2.6)$ & $1224(1.4)$ & $*$ & $146(0.8)$ \\
\hline Cemented stem & & $1600(73.6)$ & $61,377(69.1)$ & $106(25.9)$ & $5039(26.7)$ \\
\hline Cemented cup & & $1739(80.0)$ & $69,886(78.7)$ & $45(11.0)$ & $|50|(8.0)$ \\
\hline \multirow[t]{4}{*}{ Hospital } & University & $776(35.7)$ & $33,938(38.2)$ & NA & NA \\
\hline & County & $85 I(39.2)$ & $29,220(32.9)$ & NA & NA \\
\hline & Rural & $319(14.7)$ & $17,725(20.0)$ & NA & NA \\
\hline & Private & $227(10.4)$ & $7947(8.9)$ & NA & NA \\
\hline \multirow[t]{3}{*}{ Education } & Low ( $\leq 9$ years) & $453(20.8)$ & $22,660(25.5)$ & $164(40.0)$ & $6158(32.7)$ \\
\hline & Middle (9-12 years) & $814(37.5)$ & $29,323(33.0)$ & $163(39.8)$ & $8572(45.5)$ \\
\hline & High (> 12 years) & $906(4 I .7)$ & 36,847 (4I.5) & $83(20.2)$ & $4 I 24(21.9)$ \\
\hline \multirow[t]{3}{*}{ Civil status } & Married & II 34 (52.2) & $49,133(55.3)$ & $204(49.8)$ & $10,928(58.0)$ \\
\hline & Single & 647 (29.8) & $25,930(29.2)$ & $123(30.0)$ & $4516(23.9)$ \\
\hline & Widow/widower & $392(18.0)$ & 13,767 (I5.5) & $83(20.2)$ & $3410(18.1)$ \\
\hline
\end{tabular}


Table 2 (Continued).

\begin{tabular}{|c|c|c|c|c|c|}
\hline Variable & Level & $\begin{array}{r}\text { SE PJI } \\
\text { n (\%) }\end{array}$ & $\begin{array}{r}\text { SE Total } \\
\text { n (\%) }\end{array}$ & $\begin{array}{r}\text { DK PJI } \\
\text { n (\%) }\end{array}$ & $\begin{array}{r}\text { DK Total } \\
\text { n (\%) }\end{array}$ \\
\hline \multirow[t]{3}{*}{ Charlson } & 0 & $1413(65.0)$ & $67,437(75.9)$ & $328(80.0)$ & $16,132(85.6)$ \\
\hline & I & $423(19.5)$ & I2,704 (I4.3) & $63(15.4)$ & 2243 (11.9) \\
\hline & $2+$ & $337(15.5)$ & $8689(9.8)$ & $19(4.6)$ & $479(2.5)$ \\
\hline \multirow[t]{4}{*}{ Elixhauser } & 0 & $946(43.5)$ & $50,213(56.5)$ & $280(68.3)$ & $14,753(78.2)$ \\
\hline & I & $539(24.8)$ & $21,348(24.0)$ & $77(18.8)$ & $2716(14.4)$ \\
\hline & 2 & $372(17.1)$ & $10,734(12.1)$ & $35(8.5)$ & $900(4.8)$ \\
\hline & $3+$ & $316(14.5)$ & $6535(7.4)$ & $18(4.4)$ & $485(2.6)$ \\
\hline \multirow[t]{4}{*}{ RxRiskV } & $-5-0$ & $448(20.6)$ & $25,956(29.2)$ & $123(30.0)$ & $8229(43.6)$ \\
\hline & $1-3$ & $711(32.7)$ & $31,557(35.5)$ & $153(37.3)$ & $6430(34.1)$ \\
\hline & $4-6$ & $547(25.2)$ & $|9,85|(22.3)$ & $86(21.0)$ & $2770(14.7)$ \\
\hline & $7+$ & $467(21.5)$ & 11,466 (12.9) & 48 (II.7) & I 425 (7.6) \\
\hline AIDS/HIV & & I $(0.0)$ & $16(0.0)$ & $0(0.0)$ & $8(0.0)$ \\
\hline Anemia & & $20(0.9)$ & $619(0.7)$ & $0(0.0)$ & $49(0.3)$ \\
\hline Arrhythmia & & $27 \mid(12.5)$ & $6368(7.2)$ & $28(6.8)$ & $843(4.5)$ \\
\hline Arterial hypertension & & $753(34.7)$ & $24,058(27.1)$ & $45(11.0)$ & $1440(7.6)$ \\
\hline Cancer & & $142(6.5)$ & $3997(4.5)$ & $20(4.9)$ & $822(4.4)$ \\
\hline CNS disease & & $166(7.6)$ & $3142(3.5)$ & $17(4.1)$ & $310(1.6)$ \\
\hline Coagulopathy & & $9(0.4)$ & $329(0.4)$ & $0(0.0)$ & $17(0.1)$ \\
\hline Diabetes & & $212(9.8)$ & $5973(6.7)$ & $17(4.1)$ & $493(2.6)$ \\
\hline Drug alcohol abuse & & $30(1.4)$ & $682(0.8)$ & $6(1.5)$ & $105(0.6)$ \\
\hline Fluid electrolyte disorders & & $29(1.3)$ & $534(0.6)$ & $8(2.0)$ & $195(1.0)$ \\
\hline Heart condition & & $149(6.9)$ & $3693(4.2)$ & $13(3.2)$ & $421(2.2)$ \\
\hline Myocardial infarction & & $105(4.8)$ & $3087(3.5)$ & $0(0.0)$ & $73(0.4)$ \\
\hline Hypothyroidism & & $82(3.8)$ & $2583(2.9)$ & * & * \\
\hline Kidney disease & & $36(1.7)$ & $883(1.0)$ & * & $*$ \\
\hline Liver disease & & $33(1.5)$ & $524(0.6)$ & $*$ & * \\
\hline Lung airways disease & & $172(7.9)$ & $4317(4.9)$ & $20(4.9)$ & $537(2.8)$ \\
\hline Peptic ulcer & & $21(1.0)$ & $536(0.6)$ & * & $*$ \\
\hline Rheumatic disease & & 144 (6.6) & $3926(4.4)$ & $12(2.9)$ & $384(2.0)$ \\
\hline Vascular disease & & $89(4.1)$ & $2523(2.8)$ & $21(5.1)$ & $506(2.7)$ \\
\hline Weight loss & & $5(0.2)$ & $69(0.1)$ & $0(0.0)$ & $29(0.2)$ \\
\hline
\end{tabular}

Notes: *Cells have been intentionally left blank due to small numbers and privacy concerns for the Danish cohort.

Abbreviations: NA, data not available; BMI, body mass index; ASA class, American Society for Anaesthesiologists classification; CNS, central nervous system. 


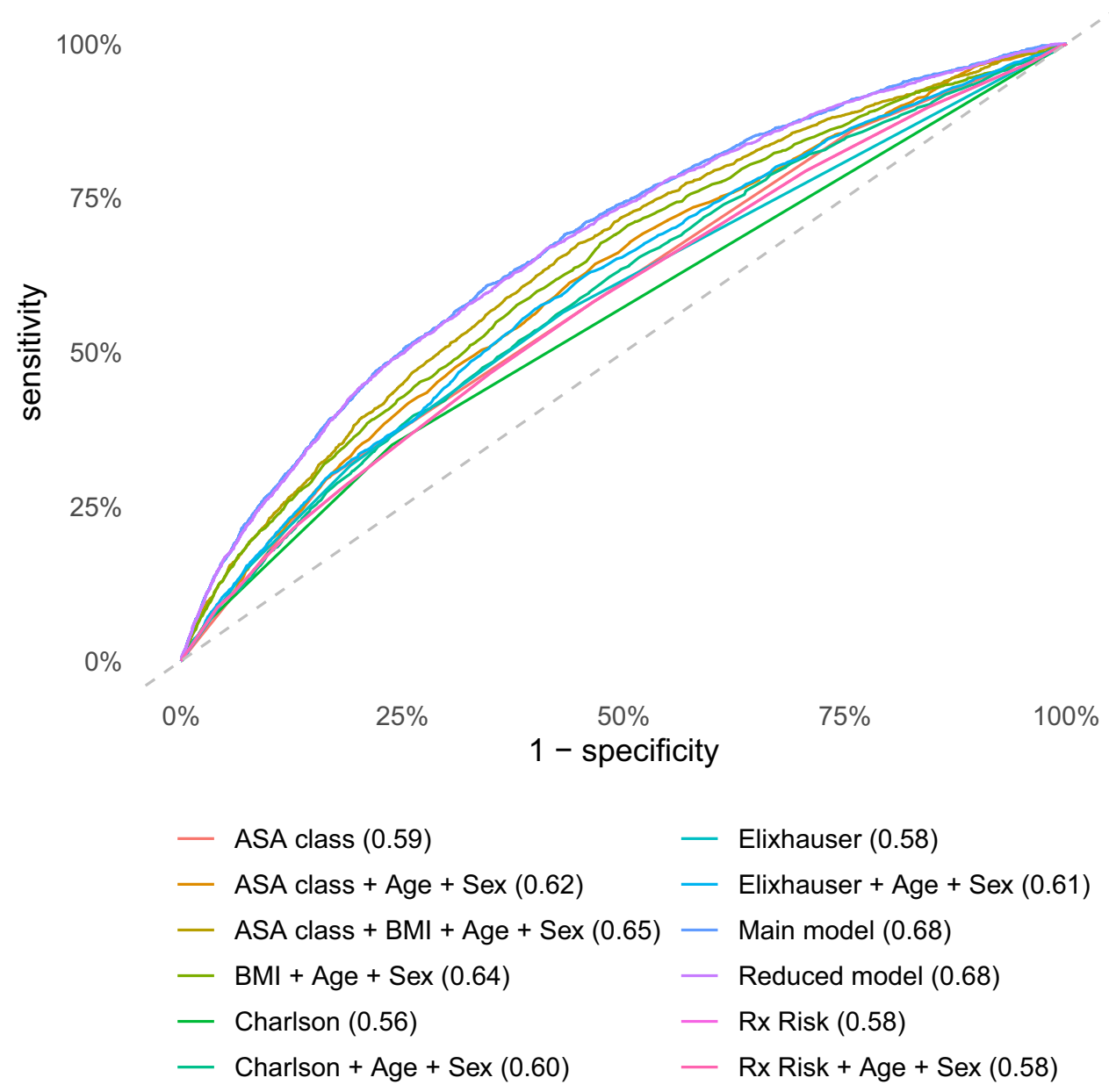

Figure 3 Receiver operating characteristics (ROC) curves combines sensitivity and specificity to illustrate discriminative abilities of the different models. The main and reduced models performed almost identical for prediction of periprosthetic joint infection (PJI) within 90 days after surgery. They both performed better than all other models. Area under the curve (AUC) are stated for each curve within parenthesis.

Abbreviations: BMI, body mass index; ASA, American Society for Anesthesiologists classification; Charlson, Charlson comorbidity index; Elixhauser, Elixhauser comorbidity index.

and without PJI were partially overlapping, but patients with PJI had on average higher predicted probabilities for this outcome (Figure 6).

The reduced model derived from the Swedish cohort produced a very similar ROC curve when applied to the Danish cohort. Calibration was good for predictions up to $3 \%$, with a tendency to over-estimate higher probabilities, which improved after re-calibration of the model intercept to the Danish PJI incidence (Figure 5).

\section{Discussion}

\section{Principal Findings}

We propose a PJI prediction model based on the underlying diagnosis for THA, BMI, ASA class, sex, age, and the presence of arrhythmia, CNS disease, fluid electrolyte disorders, liver disease or lung and airways disease. This model was better at predicting PJI within 90 days after THA than models based on either ASA class or the Charlson, Elixhauser or Rx Risk V comorbidity indices, with or without adjustments for age and sex, in terms of AUC, Nagelkerke's $R^{2}$ and calibration.

\section{Strengths and Weaknesses}

Our external model validation is a strength, supporting that the model works equally well in a different population. The validation was both geographical (different country) and temporal (different period). Discriminatory 


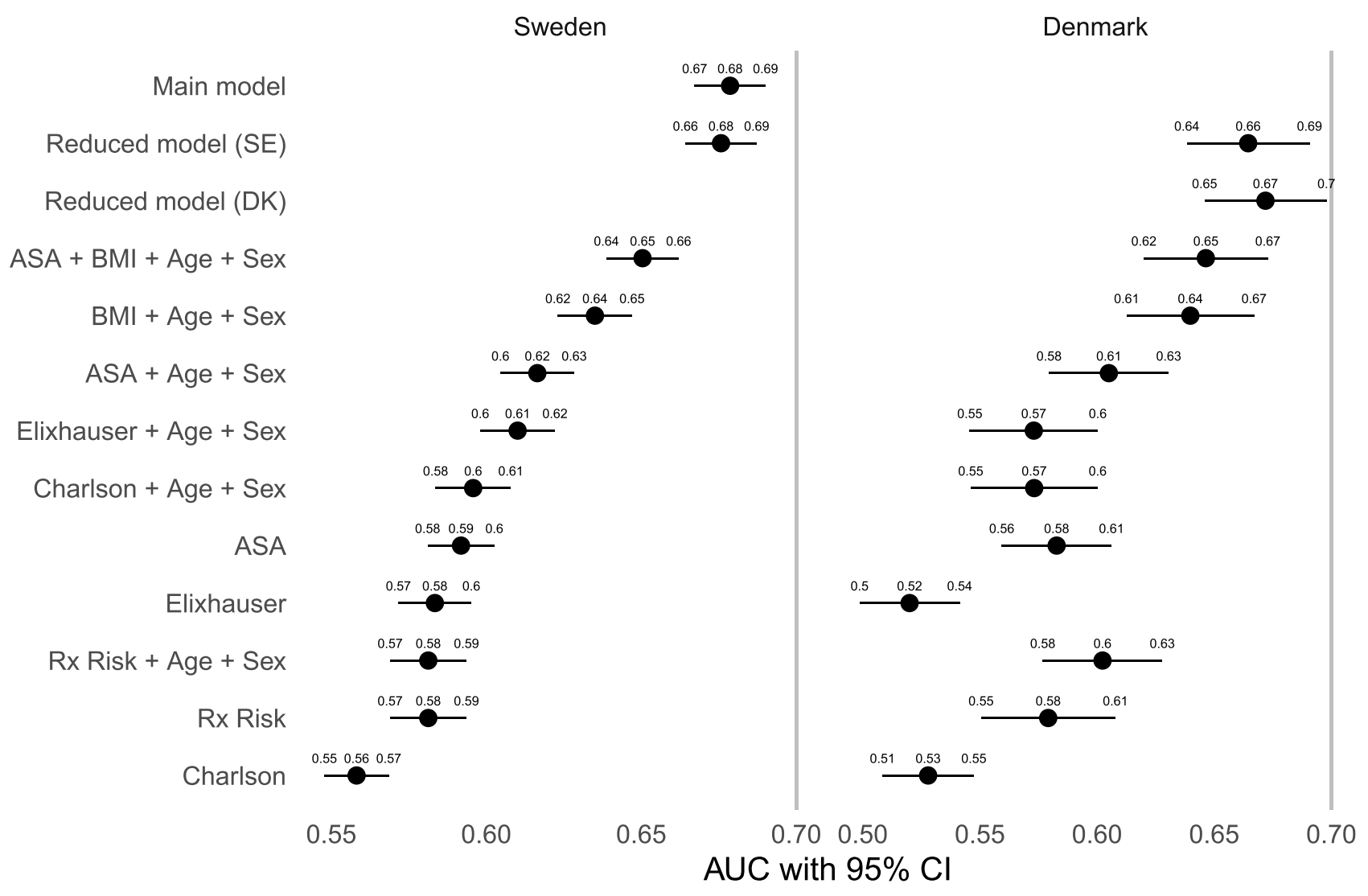

Figure 4 Area under the receiver operating characteristics curve (AUC) as a measure of predictive discriminative ability with $95 \%$ confidence intervals. The reduced model performed no different than the main model on the Swedish data, and both of these models performed better than all other models (left panel). Similar models were fitted to the Danish cohort (right panel). The reduced model (SE) with coefficients based on the Swedish data, performed almost as good as the reduced model (DK) with coefficient values refitted to the Danish cohort.

Abbreviations: BMI, body mass index; ASA, American Society for Anesthesiologists classification; Charlson, Charlson comorbidity index; Elixhauser, Elixhauser comorbidity index; DK, Denmark; SE, Sweden.

ability and calibration were good in the validation cohort despite apparent dissimilarities between the countries (Table 2). Some of the dissimilarities can partially be explained by different coding routines. ICD-10 and NOMESCO codes are recorded for both in- and outpatient hospital visits in the national patient registries in both countries, but the recording of (optional) secondary diagnoses is $23 \%$ in Denmark, ${ }^{26}$ compared to $47 \%$ in the Swedish cohort. Serious comorbidities such as cancer, are reported equally well in both countries, while for example arterial hypertension, is less well documented in Denmark. Also, patients with chronic conditions, such as diabetes, are more commonly treated in primary care settings in Denmark, and are therefore less well reported to the national patient register. ${ }^{26}$ The RxRisk V index is a weighted index, which is difficult to compare without further analysis. Taken together, the similarities between the countries might be more pronounced than indicated by Table 2. However, the difference in the mode of THA fixation, with a dominance of cemented components in Sweden, and much more widespread usage of uncemented components in Denmark, is tangible and has been described elsewhere. ${ }^{34}$

\section{Strengths and Weaknesses in Relation to Other Studies}

The standard peri-operative prophylaxis in THA surgery in Sweden is Cloxacillin (2g x 3) on the day of surgery. Those with confirmed or suspected allergy to Cloxacillin (roughly $10 \%$ of all patients) receive Clindamycin (600 $\mathrm{mg} \times 2$ ). This treatment might affect the outcome of interest, and therefore the possibility to generalize the results to countries with other treatment regimens. 
Table 3 Estimated Coefficients $(\beta)$ and Odds Ratios (OR) with 95\% Confidence Intervals for the Reduced Model

\begin{tabular}{|c|c|c|c|c|}
\hline Variable & Level & $\beta$ & OR 95 (\% Cl) & $\mathbf{p}$ \\
\hline (Intercept) & & -6.31 & & \\
\hline Age & & 0.02 & $1.02(1.02-1.03)$ & $<0.001$ \\
\hline Arrhythmia & & 0.27 & $1.30(1.13-1.49)$ & $<0.001$ \\
\hline \multirow[t]{3}{*}{ ASA class } & I & 0.00 & (ref) & \\
\hline & II & 0.18 & $1.20(1.05-1.37)$ & 0.009 \\
\hline & III & 0.44 & $1.56(1.33-1.83)$ & $<0.001$ \\
\hline \multirow[t]{4}{*}{ BMI } & Under/normal weight & 0.00 & (ref) & \\
\hline & Overweight & 0.39 & $1.47(1.32-1.65)$ & $<0.001$ \\
\hline & Class I obesity & 0.81 & $2.24(1.97-2.55)$ & $<0.001$ \\
\hline & Class II-III obesity & 1.40 & $4.05(3.46-4.75)$ & $<0.001$ \\
\hline CNS disease & & 0.69 & $2.00(1.68-2.35)$ & $<0.001$ \\
\hline \multirow[t]{5}{*}{ Diagnosis } & Primary osteoarthritis & 0.00 & (ref) & \\
\hline & Sequelae after childhood hip disease & 0.39 & $1.48(1.06-2.01)$ & 0.016 \\
\hline & Avascular necrosis of the femoral head (AVN) & 0.58 & $1.79(1.40-2.26)$ & $<0.001$ \\
\hline & Secondary osteoarthritis & 0.74 & $2.09(I .80-2.4 I)$ & $<0.001$ \\
\hline & Inflammatory joint disease & 0.94 & $2.55(1.91-3.33)$ & $<0.001$ \\
\hline Fluid electrolyte disorders & & 0.42 & $1.52(1.01-2.20)$ & 0.034 \\
\hline Liver disease & & 0.75 & $2.11(1.44-3.00)$ & $<0.001$ \\
\hline Lung airways disease & & 0.27 & $1.31(1.11-1.54)$ & 0.001 \\
\hline \multirow[t]{2}{*}{ Sex } & Female & 0.00 & (ref) & \\
\hline & Male & 0.37 & $1.45(1.33-1.59)$ & $<0.001$ \\
\hline
\end{tabular}

Abbreviations: BMI, body mass index; CNS, central nervous system; ASA, American Society for Anesthesiologists.

Cancer seems to confer an increased risk of PJI in several observational studies, ${ }^{10,19,35,36}$ but this comorbidity was not included in our model. Neither was diabetes included in our model, although it is included in previous risk calculators. ${ }^{19,37,38}$ On the other hand, diabetes is not consistently found to be associated with the risk of surgical site infection or PJI after THA surgery. ${ }^{39}$

Information on peri-operative hyperglycaemia, morning blood glucose, HBA1c, ${ }^{40,41}$ or low preoperative haemoglobin, ${ }^{12}$ could have improved our model. But although we had access to the ICD codes defining the presence of anaemia, we had no information on actual laboratory findings. Use of subcutaneously applied Tumour Necrosis Factor-alpha blockers, ${ }^{42,43}$ intra-articular steroid application ${ }^{44}$ or prolonged operating time, ${ }^{13,45,46}$ are also associated with the risk of PJI after THA. However, we decided to only consider factors that are available in an outpatient setting prior to surgery and our model can thus be used as a tool for shared decision making. This is facilitated by use of the implemented web calculator which we believe is a strength of our model. Another strength is the closed form regression formula presented below which is more transparent than a black box model sometimes associated with machine learning and artificial intelligence. 
Table 4 Model Performance Based on the Swedish Derivation Cohort

\begin{tabular}{|l|l|r|r|}
\hline Model & AUC (95\% Cl) & $\mathbf{R}^{2}(\%)$ & FNI (\%) \\
\hline Main model & $0.68(0.67 ; 0.69)$ & 4.86 & 85 \\
\hline Reduced model & $0.68(0.66 ; 0.69)$ & 4.72 & 84 \\
\hline ASA + BMI + Age + Sex & $0.65(0.64 ; 0.66)$ & 3.32 & 78 \\
\hline BMI + Age + Sex & $0.64(0.62 ; 0.65)$ & 2.75 & 73 \\
\hline ASA + Age + Sex & $0.62(0.60 ; 0.63)$ & 1.94 & 52 \\
\hline Elixhauser + Age + Sex & $0.61(0.60 ; 0.62)$ & 1.75 & 53 \\
\hline ASA & $0.59(0.58 ; 0.60)$ & 1.57 & 43 \\
\hline Elixhauser & $0.58(0.57 ; 0.60)$ & 1.29 & 42 \\
\hline Charlson + Age + Sex & $0.60(0.58 ; 0.61)$ & 1.26 & 29 \\
\hline Rx Risk & $0.58(0.57 ; 0.59)$ & 1.03 & 29 \\
\hline Rx Risk + Age + Sex & $0.58(0.57 ; 0.59)$ & 1.03 & 0 \\
\hline Charlson & $0.56(0.55 ; 0.57)$ & 0.73 & 58 \\
\hline
\end{tabular}

Notes: The area under the receiver operating characteristics curve (AUC) is a measure of discriminatory ability. Nagelkerke's pseudo-coefficient of determination $\left(R^{2}\right)$ is an estimate of the proportion of explained variance for each model (the correlation between observed and predicted values). The univariable Charlson model had the lowest $R^{2}$ and was therefore used as baseline for the fraction of new information (FNI) added by the other models.

Abbreviations: ASA, American Society for Anesthesiologists classification; BMI, body mass index; Charlson, Charlson comorbidity index; Elixhauser, Elixhauser comorbidity index.

\section{Implications of the Study}

Our model can be presented by a logistic regression formula, where the probability of PJI within 90 days is estimated by $1 /\left[1+\exp \left(6.31-\sum_{i} x_{i} \hat{\beta}_{i}\right)\right]$ where $\sum_{i} x_{i} \hat{\beta}_{i}$ indicates the sum of individual patient characteristics multiplied by the estimated coefficient values from Table 3. For example, a 60-year-old female with normal BMI, primary osteoarthritis and without comorbidities would have a probability of $1 /[1+\exp (6.31-60 \cdot 0.02)] \approx 0.7 \%$ of suffering from a PJI within 90 days. For comparison, an 85-year-old male with overweight, secondary osteoarthritis, psoriasis, dementia and an ASA class III would have a probability of $15.1 \%$. Note, however, that observed proportions above $10 \%$ were rare, and predicted probabilities above this limit are subject to extrapolation.

It should be noted that the aim of prediction is different from statistical inference. As such, CIs in Table 3 were based solely on the final logistic regression model, ignoring additional uncertainty from the preceding variable selection. Hence, those CIs should not be interpreted in support of any implicit hypothesis testing concerning the relevance of any individual variable. Also, the individual odds ratios should not be interpreted as effect measures of individual relations since some variables were correlated. The selected variables are nevertheless coherent with previous studies. Patients with primary osteoarthritis seem less prone to developing PJI than patients receiving THA for other reasons. ${ }^{10,21,47,48}$ Obesity is associated with an increased risk of re-operations or adverse events after $\mathrm{THA}^{49}$ and with an increased risk of developing surgical site infections or PJI after THA. ${ }^{7,11,13,35,37}$ CNS disease (cerebrovascular disease, dementia, hemiplegia, and Parkinson's disease) has also been previously associated with PJI, ${ }^{10}$ as have male sex, ${ }^{19,37,48}$ liver disease $^{50}$ and ASA class. ${ }^{39,46,51}$ 


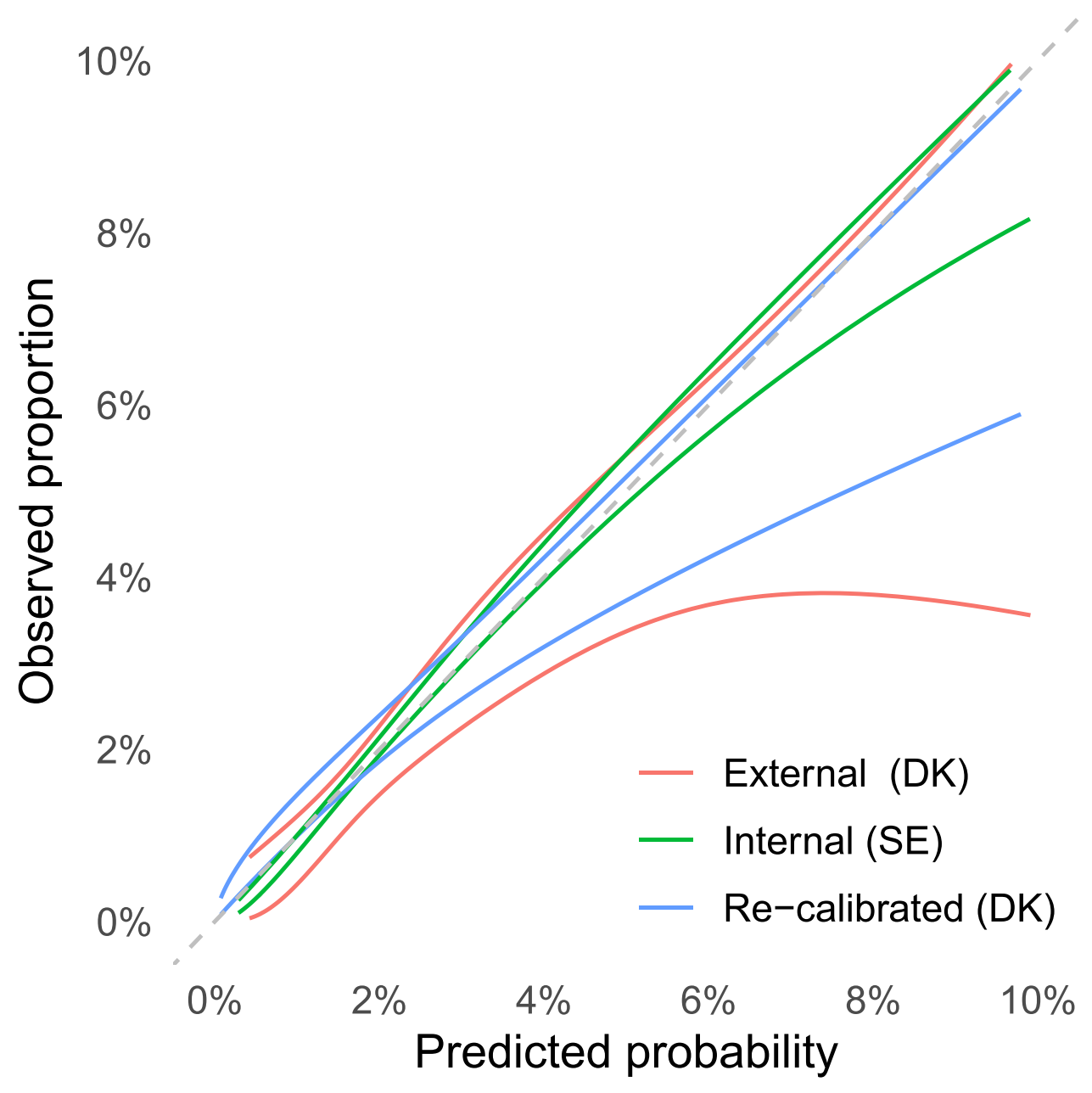

Figure 5 Calibration for the reduced model. Proportions of periprosthetic joint infection (PJI) above $10 \%$ were rarely observed and are therefore omitted. Internal calibration ( $95 \%$ confidence band between the green lines) was good; predicted probabilities were similar to observed proportions, as indicated by close proximity to the diagonal line. External calibration with the same model (red) indicated some over-estimation and less accuracy. This was expected due to smaller sample size in the Danish cohort, and due to national differences. Calibration improved after re-calibration of the model intercept to better resemble the Danish base incidence (blue). DK, Denmark; SE, Sweden.

\section{Unanswered Questions and Future Research}

The proportion of explained variation (Nagelkerke's $R^{2}=4.72 \%$ from Table 4 ) was low. The question of what other variables might be needed to further refine prediction models remains open, but information that is notoriously difficult to obtain within the setting of large register studies, such as smoking status, might be of value. In addition, a potential underlying susceptibility to PJI — as has been described for patients with osteomyelitis — could be a factor of considerable influence on the outcome.

It should also be noticed that our study design was observational, and can only yield (statistical) correlations between PJI and the included variables. Additional studies are therefore required to establish causal pathways and to identify possible modifiable risk factors. ${ }^{52}$

\section{Conclusion}

Our results indicate that the risk of early PJI after THA can be pre-operatively assessed by a parsimonious prediction model based on patient demographics and a short list of well-defined comorbidities. We hope that this model, with its accompanying web calculator, will facilitate shared decision-making between physicians and patients in need of THA. 


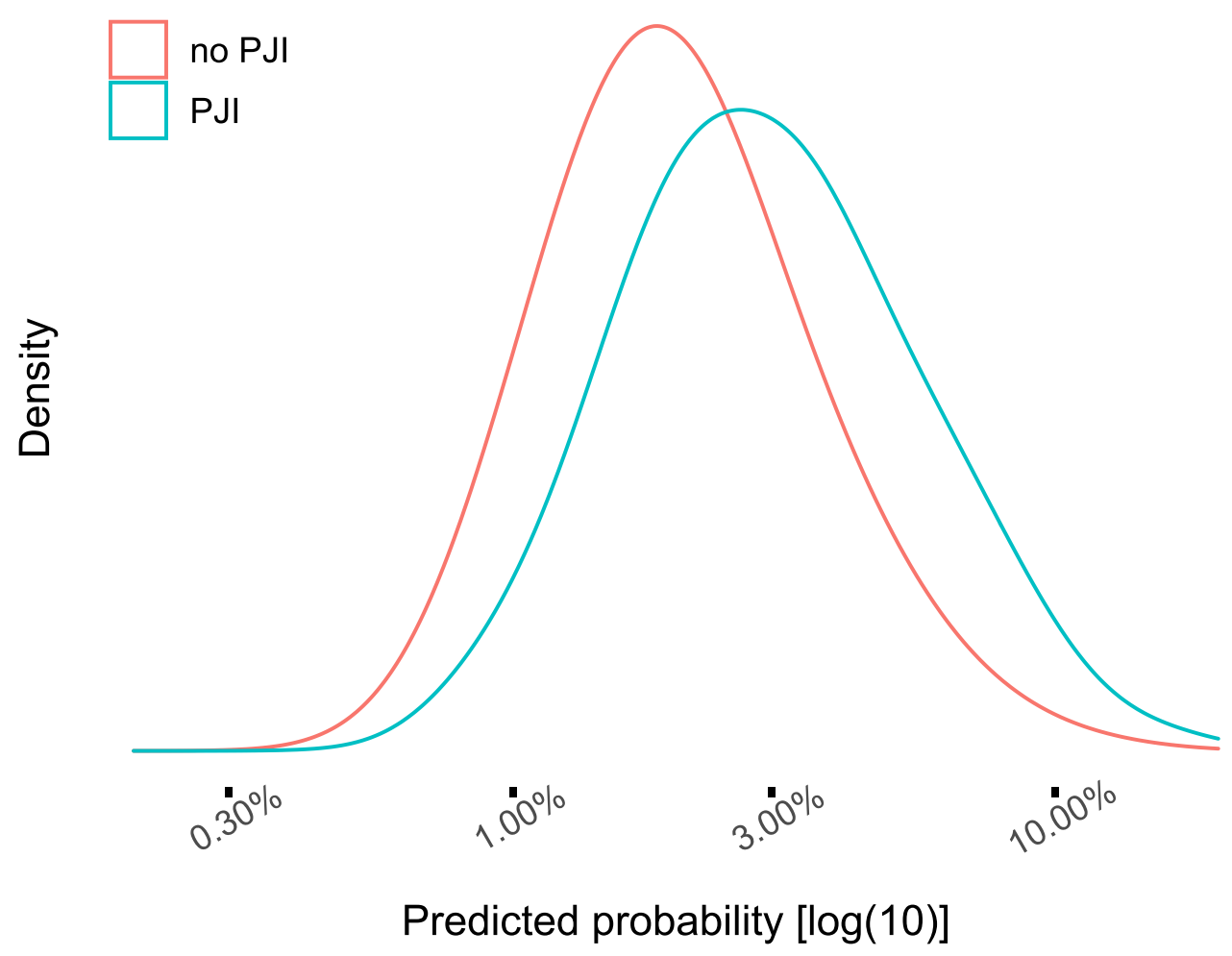

Figure 6 Patients with observed periprosthetic joint infection (PJI) had, on average, higher predicted probabilities for this adverse event, compared to patients with no PJI. The $\mathrm{x}$-axis is log-transformed for visual clarity and each curve was normalized on the $y$-axis which therefore has no direct interpretation.

\section{Abbreviations}

ASA, American Society for Anesthesiologists; AUC, area under the curve; BMI, body mass index; CI, confidence interval; DHR, The Danish Hip Arthroplasty Register; DK, Denmark; FNI, fraction of new information; ICD, International Classification of Diseases; LASSO, least absolute shrinkage and selection operator; NOMESCO, Nordic Medico-Procedural Committee; NPR, The National Patient Register; PJI, periprosthetic joint infection; ROC, receiver operating characteristic; SD, standard deviation; SE, Sweden; SHAR, The Swedish Hip Arthroplasty Register; THA, total hip arthroplasty.

\section{Data Sharing Statement}

Access to the model derivation cohort is granted after permission from the Västra Götaland Region, as the central personal data controller agency for the SHAR, in accordance with the European General Data Protection Regulation (GDPR), as well as Swedish laws and regulations (https://registercentrum.se/vara-tjaenster/datauttag/p/B1eeV66QE).

\section{Acknowledgments}

We would like to thank Aurelie Mailhac, statistician at the Department of Clinical Epidemiology at Aarhus University, for help with the external validation.

\section{Author Contributions}

All authors made a significant contribution to the work reported, whether that is in the conception, study design, execution, acquisition of data, analysis and interpretation, or in all these areas; took part in drafting, revising or critically reviewing the article; gave final approval of the version to be published; have agreed on the journal to which the article has been submitted; and agree to be accountable for all aspects of the work. 


\section{Funding}

This study was in part support by a grant to NPH from the Swedish Research Council (VR 2021-00980).

\section{Disclosure}

OR is a principal investigator for research partly founded by Pfizer; OR is a register director of the Swedish Arthroplasty Register (SAR) and board member of the International Society of Arthroplasty Registries. NPH has received institutional support from Waldemar Link GmbH, and Zimmer Biomet; also reports personal fees from Heraeus Medical, Germany. NPH is president of the Nordic Arthroplasty Register Association, member of the steering committee of SAR, and Co-Editor of Acta Orthopaedica; no other relationships or activities that could appear to have influenced the submitted work. The authors report no other conflicts of interest in this work.

\section{References}

1. Zimmerli W. Prosthetic-joint-associated infections. Best Pract Res Clin Rheumatol. 2006;20(6):1045-1063. doi:10.1016/j.berh.2006.08.003

2. Kapadia BH, Berg RA, Daley JA, Fritz J, Bhave A, Mont MA. Periprosthetic joint infection. Lancet. 2016;387(10016):386-394. doi:10.1016/ S0140-6736(14)61798-0

3. Shohat N, Bauer T, Buttaro M, et al. Hip and knee section, what is the definition of a Periprosthetic Joint Infection (PJI) of the knee and the hip? Can the same criteria be used for both joints?: proceedings of international consensus on orthopedic infections. J Arthroplasty. 2019;34(2):S325S327. doi:10.1016/j.arth.2018.09.045

4. Malizos KN, Kirketerp-Møller K. Incidence and socioeconomic impact of Bone and Joint Infections (BJIs): the European perspective. In: Kendoff D, Morgan-Jones R, Haddad FS, editors. Periprosthetic Joint Infections: Changing Paradigms. Cham: Springer International Publishing; 2016:3-18. doi:10.1007/978-3-319-30091-7_1

5. Ridgeway S, Wilson J, Charlet A, Kafatos G, Pearson A, Coello R. Infection of the surgical site after arthroplasty of the hip. J Bone Joint Surg Br. 2005;87-B(6):844-850. doi:10.1302/0301-620X.87B6.15121

6. Lai K, Bohm ER, Burnell C, Hedden DR. Presence of medical comorbidities in patients with infected primary hip or knee arthroplasties. J Arthroplasty. 2007;22(5):651-656. doi:10.1016/j.arth.2006.09.002

7. Malinzak RA, Ritter MA, Berend ME, Meding JB, Olberding EM, Davis KE. Morbidly obese, diabetic, younger, and unilateral joint arthroplasty patients have elevated total joint arthroplasty infection rates. J Arthroplasty. 2009;24(6):84-88. doi:10.1016/j.arth.2009.05.016

8. Ong KL, Kurtz SM, Lau E, Bozic KJ, Berry DJ, Parvizi J. Prosthetic joint infection risk after total hip arthroplasty in the medicare population. J Arthroplasty. 2009;24(6):105-109. doi:10.1016/j.arth.2009.04.027

9. Pedersen AB, Mehnert F, Johnsen SP, Sørensen HT. Risk of revision of a total hip replacement in patients with diabetes mellitus: a population-based follow up study. J Bone Joint Surg Br. 2010;92-B(7):929-934. doi:10.1302/0301-620X.92B7.24461

10. Bozic KJ, Lau E, Kurtz S, et al. Patient-related risk factors for periprosthetic joint infection and postoperative mortality following total hip arthroplasty in medicare patients. J Bone Joint Surg. 2012;94(9):794-800. doi:10.2106/JBJS.K.00072

11. Jämsen E, Nevalainen P, Eskelinen A, Huotari K, Kalliovalkama J, Moilanen T. Obesity, diabetes, and preoperative hyperglycemia as predictors of periprosthetic joint infection: a single-center analysis of 7181 primary hip and knee replacements for osteoarthritis. J Bone Joint Surg Am. 2012;94 (14):e101-1-9. doi:10.2106/JBJS.J.01935

12. Rasouli MR, Restrepo C, Maltenfort MG, Purtill JJ, Parvizi J. Risk factors for surgical site infection following total joint arthroplasty. J Bone Joint Surg Am. 2014;96(18):e158-1-5. doi:10.2106/JBJS.M.01363

13. Maoz G, Phillips M, Bosco J, et al. The Otto Aufranc award: modifiable versus nonmodifiable risk factors for infection after hip arthroplasty. Clin Orthop Relat Res. 2015;473(2):453-459. doi:10.1007/s11999-014-3780-x

14. Lespasio M, Mont M, Guarino A. Identifying risk factors associated with postoperative infection following elective lower-extremity total joint arthroplasty. Perm J. 2020;24:20.013. doi:10.7812/TPP/20.013

15. Ren X, Ling L, Qi L, et al. Patients' risk factors for periprosthetic joint infection in primary total hip arthroplasty: a meta-analysis of 40 studies. BMC Musculoskelet Disord. 2021;22(1):776. doi:10.1186/s12891-021-04647-1

16. Resende VAC, Neto AC, Nunes C, Andrade R, Espregueira-Mendes J, Lopes S. Higher age, female gender, osteoarthritis and blood transfusion protect against periprosthetic joint infection in total hip or knee arthroplasties: a systematic review and meta-analysis. Knee Surg Sports Traumatol Arthrosc. 2021;29(1):8-43. doi:10.1007/s00167-018-5231-9

17. Sodhi N, Anis HK, Vakharia RM, et al. What are risk factors for infection after primary or revision total joint arthroplasty in patients older than 80 years? Clin Orthop Relat Res. 2020;478(8):1741-1751. doi:10.1097/CORR.0000000000001389

18. Edelstein AI, Kwasny MJ, Suleiman LI, et al. Can the American college of surgeons risk calculator predict 30-day complications after knee and hip arthroplasty? J Arthroplasty. 2015;30(9):5-10. doi:10.1016/j.arth.2015.01.057

19. Wingert NC, Gotoff J, Parrilla E, Gotoff R, Hou L, Ghanem E. The ACS NSQIP risk calculator is a fair predictor of acute periprosthetic joint infection. Clin Orthop Relat Res. 2016;474(7):1643-1648. doi:10.1007/s11999-016-4717-3

20. Bozic KJ, Ong K, Lau E, et al. Estimating risk in medicare patients with THA: an electronic risk calculator for periprosthetic joint infection and mortality. Clin Orthop Relat Res. 2013;471(2):574-583. doi:10.1007/s11999-012-2605-z

21. Inacio MCS, Pratt NL, Roughead EE, Graves SE. Predicting infections after total joint arthroplasty using a prescription based comorbidity measure. J Arthroplasty. 2015;30(10):1692-1698. doi:10.1016/j.arth.2015.05.004

22. Cnudde P, Rolfson O, Nemes S, et al. Linking Swedish health data registers to establish a research database and a shared decision-making tool in hip replacement. BMC Musculoskelet Disord. 2016;17(1):414. doi:10.1186/s12891-016-1262-x

23. Bülow E, Nemes S, Rolfson O. Are the first or the second hips of staged bilateral THAs more similar to unilateral procedures? A study from the Swedish hip arthroplasty register. Clin Orthop Relat Res. 2020;2020(478):11262-11270. doi:10.1097/CORR.0000000000001210 
24. Schmidt M, Pedersen L, Sørensen HT. The Danish Civil Registration System as a tool in epidemiology. Eur J Epidemiol. 2014;29(8):541-549. doi:10.1007/s10654-014-9930-3

25. Gundtoft PH, Varnum C, Pedersen AB, Overgaard S. The Danish hip arthroplasty register. CLEP. 2016;8:509-514. doi:10.2147/CLEP.S99498

26. Schmidt M, Schmidt SAJ, Sandegaard JL, Ehrenstein V, Pedersen L, Sørensen HT. The Danish National Patient Registry: a review of content, data quality, and research potential. Clin Epidemiol. 2015;7:449-490. doi:10.2147/CLEP.S91125

27. Data for research. Available from: https://www.dst.dk/en/TilSalg/Forskningsservice. Accessed June 17, 2021.

28. Garland A, Bülow E, Lenguerrand E, et al. Prediction of 90-day mortality after total hip arthroplasty. Bone Joint J. 2021;103-B(3):469-478. doi:10.1302/0301-620X.103B3.BJJ-2020-1249.R1

29. Kildemoes HW, Sørensen HT, Hallas J. The Danish national prescription registry. Scand J Public Health. 2011;39(7 Suppl):38-41. doi:10.1177/ 1403494810394717

30. Pratt NL, Kerr M, Barratt JD, et al. The validity of the Rx-Risk comorbidity index using medicines mapped to the anatomical therapeutic chemical (ATC) classification system. BMJ Open. 2018;8(4):e021122. doi:10.1136/bmjopen-2017-021122

31. Nattino G, Finazzi S, Bertolini G. A new test and graphical tool to assess the goodness of fit of logistic regression models. Stat Med. 2016;35 (5):709-720. doi:10.1002/sim.6744

32. Harrell F. Statistically efficient ways to quantify added predictive value of new measurements. Statistical thinking; 2018. Available from: https:// www.fharrell.com/post/addvalue/. Accessed May 11, 2021.

33. Bülow E. Coder: an R package for code-based item classification and categorization. J Open Source Softw. 2020;5(56):2916. doi:10.21105/ joss.02916

34. Bunyoz KI, Malchau E, Malchau H, Troelsen A. Has the use of fixation techniques in THA changed in this decade? The uncemented paradox revisited. Clin Orthop Relat Res. 2020;478(4):697-704. doi:10.1097/CORR.0000000000001117

35. Everhart JS, Altneu E, Calhoun JH. Medical comorbidities are independent preoperative risk factors for surgical infection after total joint arthroplasty. Clin Orthop Relat Res. 2013;471(10):3112-3119. doi:10.1007/s11999-013-2923-9

36. Poultsides LA, Ma Y, Della Valle AG, Chiu YL, Sculco TP, Memtsoudis SG. In-hospital surgical site infections after primary hip and knee arthroplasty incidence and risk factors. J Arthroplasty. 2013;28(3):385-389. doi:10.1016/j.arth.2012.06.027

37. Tan TL, Maltenfort MG, Chen AF, et al. Development and evaluation of a preoperative risk calculator for periprosthetic joint infection following total joint arthroplasty. J Bone Joint Surg. 2018;100:777-785. doi:10.2106/JBJS.16.01435

38. Inacio MCS, Pratt NL, Roughead EE, Graves SE. Comparing co-morbidities in total joint arthroplasty patients using the RxRisk-V, Elixhauser, and Charlson Measures: a cross-sectional evaluation. BMC Musculoskelet Disord. 2015;16(1):385. doi:10.1186/s12891-015-0835-4

39. Namba RS, Inacio MCS, Paxton EW. Risk factors associated with surgical site infection in 30,491 primary total hip replacements. $J$ Bone Joint Surg Br. 2012;94-B(10):1330-1338. doi:10.1302/0301-620X.94B10.29184

40. Mraovic B, Suh D, Jacovides C, Parvizi J. Perioperative hyperglycemia and postoperative infection after lower limb arthroplasty. J Diabetes Sci Technol. 2011;5(2):412-418. doi:10.1177/193229681100500231

41. Iorio R, Williams KM, Marcantonio AJ, Specht LM, Tilzey JF, Healy WL. Diabetes mellitus, hemoglobin A1C, and the incidence of total joint arthroplasty infection. J Arthroplasty. 2012;27(5):726-729.e1. doi:10.1016/j.arth.2011.09.013

42. Gilson M, Gossec L, Mariette X, et al. Risk factors for total joint arthroplasty infection in patients receiving tumor necrosis factor -blockers: a case-control study. Arthritis Res Ther. 2010;12(4):R145. doi:10.1186/ar3087

43. Momohara S, Kawakami K, Iwamoto T, et al. Prosthetic joint infection after total hip or knee arthroplasty in rheumatoid arthritis patients treated with nonbiologic and biologic disease-modifying antirheumatic drugs. Mod Rheumatol. 2011;21(5):469-475. doi:10.3109/s10165-011-0423-x

44. Cancienne JM, Werner BC, Luetkemeyer LM, Browne JA. Does timing of previous intra-articular steroid injection affect the post-operative rate of infection in total knee arthroplasty? J Arthroplasty. 2015;30(11):1879-1882. doi:10.1016/j.arth.2015.05.027

45. Willis-Owen CA, Konyves A, Martin DK. Factors affecting the incidence of infection in hip and knee replacement: an analysis of 5277 cases. J Bone Joint Surg Br. 2010;92-B(8):1128-1133. doi:10.1302/0301-620X.92B8.24333

46. Berbari EF, Osmon DR, Lahr B, et al. The mayo prosthetic joint infection risk score: implication for surgical site infection reporting and risk stratification. Infect Control Hosp Epidemiol. 2012;33(8):774-781. doi:10.1086/666641

47. Pedersen AB, Svendsson JE, Johnsen SP, Riis A, Overgaard S. Risk factors for revision due to infection after primary total hip arthroplasty: a population-based study of 80,756 primary procedures in the Danish Hip Arthroplasty Registry. Acta Orthopaed. 2010;81(5):542-547. doi:10.3109/ 17453674.2010 .519908

48. Dale H, Fenstad AM, Hallan G, et al. Increasing risk of prosthetic joint infection after total hip arthroplasty: 2778 revisions due to infection after 432,168 primary THAs in the Nordic Arthroplasty Register Association (NARA). Acta Orthopaed. 2012;83(5):449-458. doi:10.3109/ 17453674.2012.733918

49. Sayed-Noor AS, Mukka S, Mohaddes M, Kärrholm J, Rolfson O. Body mass index is associated with risk of reoperation and revision after primary total hip arthroplasty: a study of the Swedish Hip Arthroplasty Register including 83,146 patients. Acta Orthopaed. 2019;90(3):220-225. doi:10.1080/17453674.2019.1594015

50. Deleuran T, Vilstrup H, Overgaard S, Jepsen P. Cirrhosis patients have increased risk of complications after hip or knee arthroplasty: a Danish population-based cohort study. Acta Orthopaed. 2015;86(1):108-113. doi:10.3109/17453674.2014.961397

51. Pulido L, Ghanem E, Joshi A, Purtill JJ, Parvizi J. Periprosthetic joint infection: the incidence, timing, and predisposing factors. Clin Orthop Relat Res. 2008;466(7):1710-1715. doi:10.1007/s11999-008-0209-4

52. de Mast J, Steiner SH, Nuijten WPM, Kapitan D. Analytical problem solving based on causal, correlational and deductive models. Am Stat. 2022;1-19. doi:10.1080/00031305.2021.2023633 


\section{Publish your work in this journal}

Clinical Epidemiology is an international, peer-reviewed, open access, online journal focusing on disease and drug epidemiology, identification of risk factors and screening procedures to develop optimal preventative initiatives and programs. Specific topics include: diagnosis, prognosis, treatment, screening, prevention, risk factor modification, systematic reviews, risk \& safety of medical interventions, epidemiology \& biostatistical methods, and evaluation of guidelines, translational medicine, health policies \& economic evaluations. The manuscript management system is completely online and includes a very quick and fair peer-review system, which is all easy to use.

Submit your manuscript here: https://www.dovepress.com/clinical-epidemiology-journal 